\title{
Light-Addressable Potentiometric Sensors in Microfluidics
}

\author{
Li Xueliang ${ }^{1 *}$, Liu Shibin², Tan Jie ${ }^{2}$, Wu Chunsheng ${ }^{3 *}$ \\ (1. School of Mechanical and Electrical Engineering, Zhoukou Normal University, Zhoukou 466001, China; 2. College of \\ Electronics and Information, Northwestern Polytechnical University, Xi'an 710072, China; 3.Institute of Medical \\ Engineering, Department of Biophysics, School of Basic Medical Sciences, Health Science Center, Xi'an Jiaotong \\ University, Xi'an, 710061, China) \\ * Correspondence: zhf870721@zknu.edu.cn (Li xueliang); wuchunsheng@xjtu.edu.cn (Wu Chunsheng)
}

\begin{abstract}
Light-addressable potentiometric sensor (LAPS) is an electrochemical sensor based on the field-effect principle of semiconductor. It is able to sense the change of Nernst potential on the sensor surface, and the measuring area can be controlled by the illumination. Due to the unique light-addressable ability of LAPS, the chemical imaging system constructed with LAPS can realize the two-dimensional image distribution detection of chemical/biomass. In this paper, the advantages of LAPS as sensing unit of microelectrochemical analysis system are summarized. Then, the greatest development of LAPS analysis system is explained and discussed. Especially, this paper focused on the research of ion diffusion, enzymatic reaction, microbial metabolism and droplet microfluidics by using LAPS analysis system. Finally, the development trends and prospects of LAPS analysis system are illustrated.
\end{abstract}

Keywords: Light-Addressable Potentiometric Sensor (LAPS); Micropump; Microfluidics; Chemical Sensors; Biosensors

\section{Introduction}

Microfluidic chip technology is an emerging and powerful analysis technology, which has been widely applied in the development of microanalysis systems. That is, based on the micro-electro-mechanical system (MEMS) technology, a variety of micro-structures such as microchannels, micro-mixers, micro-reaction chambers, and micro-pumps are fabricated on glass, silicon wafers, organic polymers and other materials. A series of discontinuous processes in biochemical analysis, including sample collection, processing, reaction and detection are integrated on a chip to achieve miniaturization and integration of analysis equipment, which realizes lab-on-a-chip. Therefore, it has the advantages of fast analysis speed, less pollution, parallel processing, etc., and has gradually attracted attention and has been applied in many fields such as clinical examination and environmental monitoring ${ }^{[1]}$.

Detector is one of the core components of microfluidic chip. Since the advent of microfluidic chips, the research of detectors suitable for integration with microfluidic chips has been a hot spot. There are more than a dozen microfluidic chip detection technologies, among which light detection and electrochemical detection are the most widely used technologies. The light detection method has the advantages of high sensitivity and strong selectivity. However, fluorescent labeling not only has side effects on analysis, but also has complex structure and large volume of light detection system, making it difficult to miniaturize the microfluidics as a whole. Due to its simple structure and small size, various electrochemical detectors have incomparable advantages in integration with chips. Especially, semiconductor electrochemical sensors are compatible with micromachining technologies ${ }^{[2]}$. Notably, the electrochemical sensor-light addressed potentiometric sensor (LAPS), which is developed based on the lightelectric effect and field-effect principle in semiconductors.

LAPS is a kind of potentiometric semiconductor chemical/biological sensor, which appeared in the late 1980s. It has always been one of the research hotspots in chemical/biological sensor because of its small size, high detection accuracy, flexible use and strong addressable detection capability. In 1988, Hafeman et al. analyzed the working principle of LAPS for the first time in Science, which pointed out that LAPS can sensitively detect the weak change of solid-liquid interface potential on the surface of LAPS ${ }^{[3]}$. Therefore, various biochemical reactions such as enzymatic reaction, redox reaction, antigen-antibody specific reaction, etc., could potentially be detected by LAPS. At present, LAPS has broad application prospects in the fields of $\mathrm{pH}$ detection ${ }^{[4]}$, ion detection ${ }^{[5-6]}$, molecular detection ${ }^{[7-8]}$, enzymatic reaction ${ }^{[9]}$, cell metabolism ${ }^{[10-14]}$, immunoassay and DNA detection ${ }^{[15-17]}$. 
LAPS is an electrochemical sensor based on semiconductor field-effect theory, which has a typical electrolyteinsulator-semiconductor (EIS) structure and is mainly used to detect various chemical/biological parameters or the plane distribution of some chemical/biological parameters. LAPS is not only easy to integrate and miniaturize, but also can realize label-free and quantitative detection, thus avoiding the side effects of fluorescent reagents on the analytical solutions ${ }^{[18]}$. The surface of LAPS is flat and uniform, and its surface material has the same properties with preparing microchannels. Therefore, it is possible to construct microfluidics with arbitrary shapes on the surface of LAPS $^{[19]}$. LAPS is light-addressable, this unique quality makes it possible to detect the chemical/biological information of analytical samples at any position of the microchannels. Moreover, the materials used to prepare the microchannel paths are generally transparent, so the microfluidics constructed by LAPS as a detection sensor can also be utilized to study some chemical/biological reactions with light characteristics under a light microscope ${ }^{[20]}$. In conclusion, LAPS is very suitable as a microfluidics detection sensor. As shown in Figure 1, the decisive advantages of LAPS-based microchannel analysis system include: (1) LAPS can customize the position of sensors with its unique light-addressable function, which enhances the flexibility of microfluidic chip design, and (2) it can track and detect the time-varying information of the concentration of the analytical solution at any point (for example, the red points in figure1) on the microchannel path. With these unique advantages, LAPS has quickly become a research hotspot in the field of microfluidics.

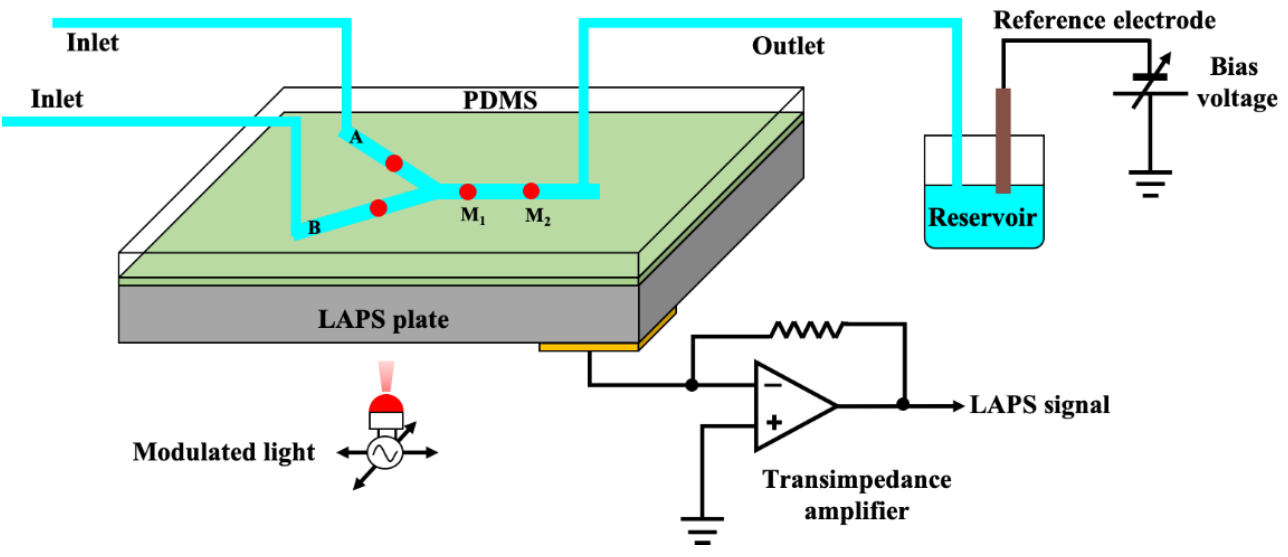

Figure 1 Schematic diagram of microchannel analysis system based on LAPS

This paper mainly summarizes the latest research progress of LAPS analysis system. In the second part, the principle of LAPS and the chemical imaging principle of LPAS are briefly introduced. The third part summarizes and discusses several applications of LAPS analysis system. Finally, the future development trends and prospects of LAPS analysis system are illustrated.

\section{Principle}

\subsection{Principle of LAPS}

The basic structure of LAPS includes four layers from top to bottom: sensing layers, insulating layer, semiconductor substrate and working electrode. Figure 2.(a) shows the structure and working principle diagram of LAPS. In the measurement, taking n-type silicon as an example, a bias voltage is applied to LAPS chip through reference electrode and working electrode. By conducting electrolyte solution, the bias voltage acts on the surface of LAPS, pushing the majority carriers at the interface between the insulating layer and the silicon substrate to the silicon substrate. Therefore, a space charge region consisting of immobile fixed ions, namely a depletion layer, is formed at the interface between the insulating layer and the silicon substrate. When LAPS is illuminated from the back by an AC modulated light source with a specific wavelength, a large number of photo-generated carriers are excited in silicon substrate. Then most of the photo-generated carriers diffuse to the depletion layer continuously. Furthermore, in the process of diffusion, some photo-generated minority carriers recombine with majority carriers. While some unbound photo-generated carriers diffuse into the depletion layer, and they are immediately separated by the internal electric field of the depletion layer. As the photo-generated minority carriers accumulate at the 
interface between the insulating layer and silicon substrate, the photo-generated potential has been formed. Since the intensity of the light source is AC modulated, the number of photo-generated minority carriers accumulated at the interface between the insulating layer and the silicon substrate also changes dynamically. The variation of photogenerated potential at the interface is synchronized with the modulation signal of the light source, forming an alternating current in an external measuring circuit. It can be detected by a galvanometer. The number of photogenerated carriers that can diffuse into the depletion layer is very small, so the amplitude of the output weak current signal ranges from tens of nanoamps to tens of microamps ${ }^{[2]}$.

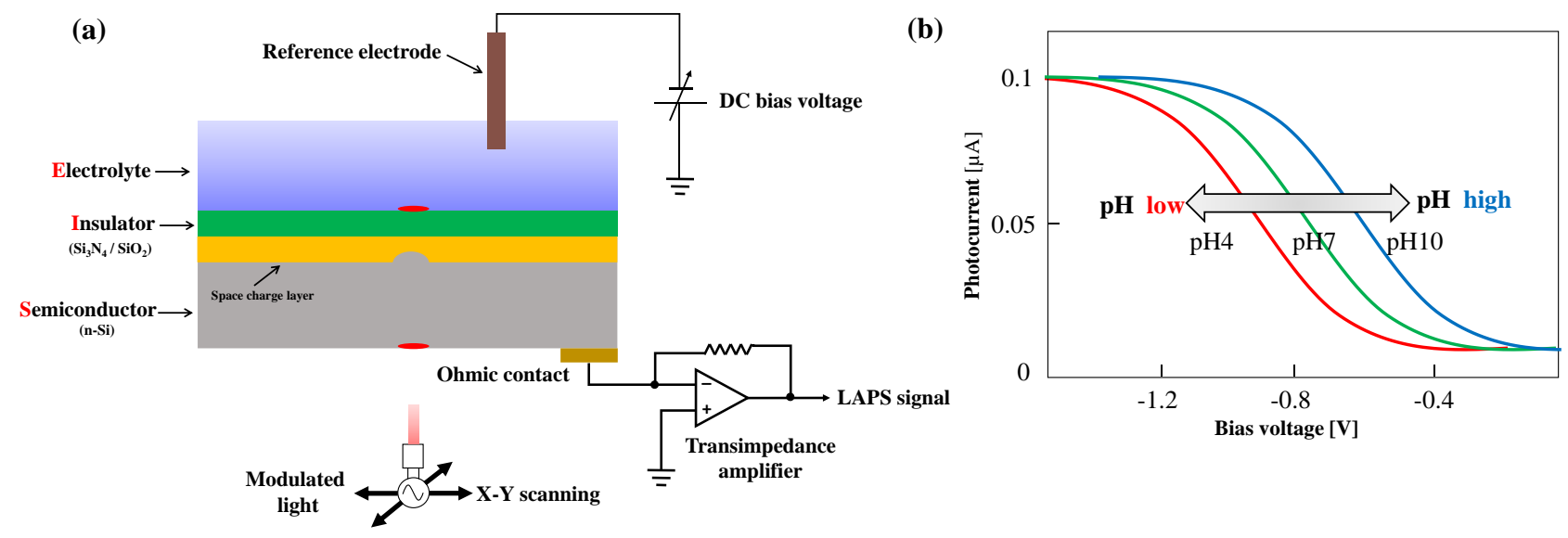

Figure 2.(a) Schematic of a typical LAPS set-up; (b) I-V characteristic curve of LAPS

In order to detect the ideal target species, it is necessary to modify the LAPS surface with a sensitive film. The sensitive film is able to generate responsive changes when the target species appears on the sensor surface. For example, the silicon nitride film which is sensitive to hydrogen ions, are commonly used to modify the LAPS surface to detect hydrogen ions. Basically, by chemical or physical methods, a sensitive film which has potential response to the species which to be measured in the electrolyte solution will deposit on the surface of LAPS insulating layer. In this case, on the surface of the sensitive film, a solid-liquid interface would be formed, it is potential proportional to the concentration of the measured species in the solution. This potential is superimposed on the external bias voltage, which changes the thickness of the space charge region between the LAPS insulating layer and the silicon substrate, which makes the change of photo-generated potential under the same light source conditions, and consequently cause the change of the current in the external measuring circuit. As a result, by recording the changes of the current amplitude, the changes in the concentrations of the measured species in the solution can be measured. Generally, the bias voltage varies linearly within a certain range, and measures the output photocurrent of LAPS at the same time. The plotted photocurrent-bias voltage curve is the I-V characteristic of LAPS, as shown in Figure 2.(b).

\subsection{Chemical imaging principle of LAPS}

LAPS can customize the detection area by changing the illumination position of the light source, so that the $\mathrm{pH}$ value of any detection point can be measured by light scanning technology. Based on the light addressable ability of LAPS, Nakao et al. proposed a chemical image sensor based on LAPS, and obtained the two-dimensional pH chemical image of the solution through this method ${ }^{[21]}$. And it was used to observe the distribution of hydrogen ions in the external microenvironment of living cells. According to the basic principle of LAPS, the pH value of solution can be obtained by the offset of $I-V$ characteristic curve. With the decrease of $\mathrm{pH}$ value, the output photocurrent of LAPS decreases as well. On the other hand, with the increase of $\mathrm{pH}$ value, the output photocurrent of LAPS also changes accordingly. When the bias voltage is fixed, the detection point of substrate of LAPS is illuminated by a light source with a certain illumination area. Therefore, the $\mathrm{pH}$ value of the illumination position can be obtained. Then, the $\mathrm{pH}$ value is converted to a gray value corresponding to the image. According to this method, the remaining positions of the substrate of LAPS are irradiated in a certain order, and finally the chemical distribution image of solution $\mathrm{pH}$ value can be obtained ${ }^{[22]}$. 


\section{Research progress of LAPS in microelectrochemical analysis system}

Since the LAPS has the decisive advantages of light addressability, flat surface, and simple structure, it has been widely applied in the development of microanalysis system via the integration with microfluidic chip. More and more attentions have been paid to this field, and some exciting progress has been made. This paper will focus on the most important and representative examples of LAPS application in microanalysis systems. Therefore, this section summarizes and discusses the applications of LAPS-based microanalysis systems, including ion diffusion, enzymatic reaction, microbial metabolic activity and droplet microfluidics.

\subsection{Ion diffusion}

The most important characteristic of LAPS is its ion sensing capability. By combining the light addressability of LAPS, an image showing the diffusion of ions on the surface of LAPS can be obtained. For instance, Yoshinobu et al. reported a miniature LAPS analysis system for studying ion diffusion ${ }^{[23]}$. Through the electrolysis of low concentration sodium chloride solution, the ion diffusion between cathode and anode was observed using this microanalysis system. This LAPS analysis system was also used to collect the acid-base concentration distribution images around the electrode, and fit the diffusion equation, calculate the diffusion coefficient and the molecular weight.

Furthermore, the integration of LAPS chip with microfluidic chip allows to monitor the dynamic changes of ions inside the microchannels. Microfluidic chip can precisely control the movement of a small amount of ion solutions inside microchannels, while LAPS can sensitively detect the concentration of local ions inside microchannels. Therefore, the combination of microfluidic chip with LAPS chip provides an ideal platform for the study of ion diffusion. Yoshinobu et al. constructed a grating PDMS microchannel on the surface of LAPS by using soft lithography technology, and built a LAPS analysis system by driving the analysis sample with a micro injector. It can observe the chemical images of some special ions or macromolecules ${ }^{[24]}$. The flow of $\mathrm{pH}$ buffer solution in the microchannels can be observed by the microelectrochemical analysis system.

With the further development and modification on microchannels, the LAPS-based microanalysis system can also be used to investigate the mechanisms of microfluidics. Miyamoto et al. constructed a LAPS analysis system with Y-shaped microchannels structure, and observed the laminar flow phenomenon in the microchannels ${ }^{[25]}$. By studying the ion diffusion law at the interface of two microchannels, the ion diffusion coefficient was determined. The photocurrent distribution of laminar flow in Y-shaped microchannels under different injection flow rates was studied. Therefore, the LAPS analysis system is expected to become a new microchannel analysis platform to study the laminar flow in microfluidics.

The feasibility of constructing microchannel analysis system on LAPS surface is demonstrated. For example, Wagner et al. expounded the advantages of constructing LAPS as detection sensor to build a microelectrochemical analysis system ${ }^{[19]}$. By constructing a Y-shaped microchannel unit on the surface of LAPS sensor, the red area represents the measurement point defined by LAPS sensor, points A and B represent the initial measurement point of two solutions respectively, P (0) represents the measurement point when the two solutions are mixed. P (1) represents the measurement point after two solutions are mixed for a period of time. All measurement points can be freely selected during operation. By attaching a layer of transparent polymer material to the local area of LAPS surface, the electrical impedance of the detection loop is increased, so as to reduce the detection photocurrent. As can be seen from the legend, the additional part of the image is obviously dimmed. Therefore, it is proved that the scheme of constructing microchannel analysis system with LAPS as detection sensor is feasible.

One of the decisive advantages of microfluidic chips originated from the large-scale of its identical units, which makes it possible to achieve high-throughput measurement. For example, Miyamoto et al. reported a LAPS analysis system which can observe the ion concentration distribution in the microchannels ${ }^{[26]}$. As shown in Figure 3, by constructing a PDMS microchannel on the surface of LAPS, and using 64 guiding fibers with different frequencies as modulated light sources, the parallel measurement of 64 detection sites was realized. Furthermore, the microfluidics was by a syringe pump, and the maximum acquisition speed of ion concentration image was 100 frames/s. The research results show that the system is expected to be widely used in the field of flow injection 
analysis, especially in the high-throughput analysis of micro droplets. This is an excellent work to demonstrate the fast-imaging speed up to now, which still have the high complexity of instrumentation from multi-frequency modulation and FFT signal process.

(a)

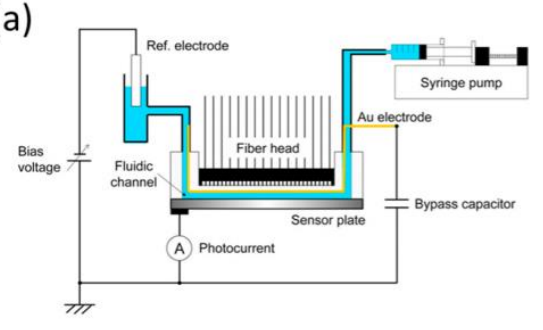

(b)

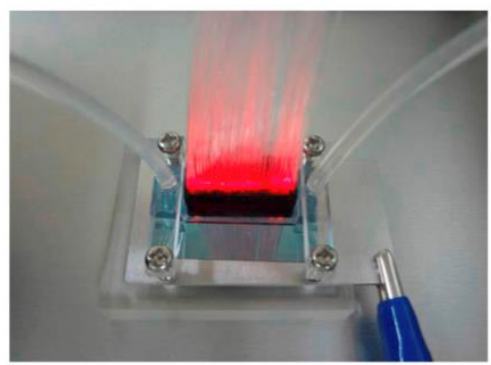

(c)

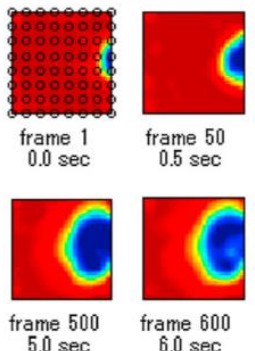

(d)
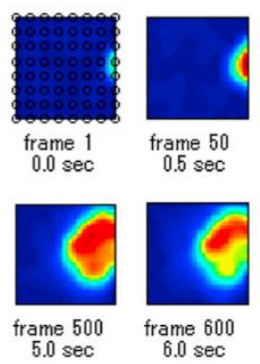
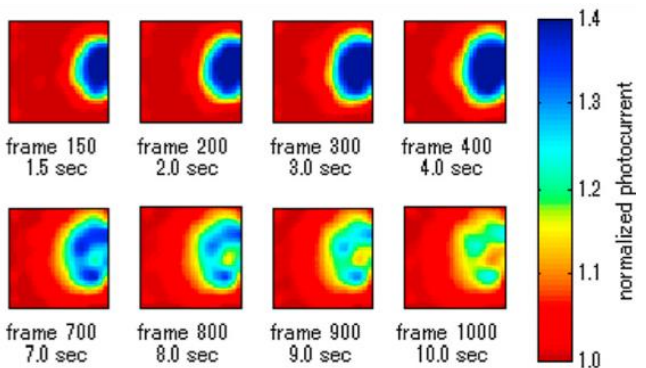

$9.0 \mathrm{sec}$

$10.0 \mathrm{sec}$
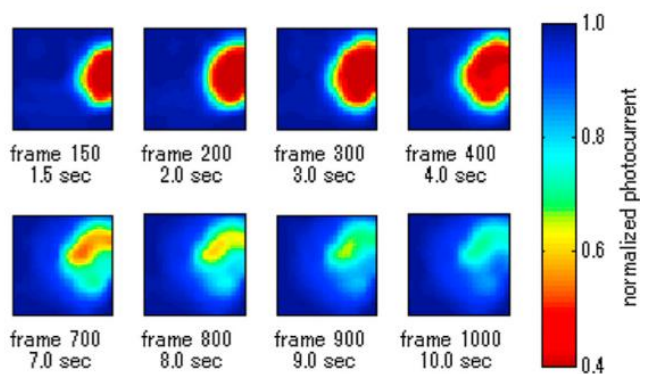

Figure 3.(a) A schematic diagram of a LAPS-based microfluidics; (b) Illumination of the front of the LAPS with 64-channel light fiber; (c) and (d) represent chemical images of injected $10 \mathrm{mM} \mathrm{NaOH}$ solution and $10 \mathrm{mM} \mathrm{HCl} \mathrm{solution}$, respectively ${ }^{[26]}$

\subsection{Enzymatic reaction}

What's more, the LAPS analysis system also shows broad application prospects in the field of biosensors, especially in the detection of enzymatic reaction products. For instance, Miyamoto et al. constructed a LAPS analysis system for detecting urea reaction products ${ }^{[27]}$. Due to the implantation of urease gel microspheres into microchannels, when the urea solution is injected into the microchannels, the $\mathrm{pH}$ change of the downstream solution of microchannels can be detected, which can reflect the kinetic process of enzymatic reaction in the microchannels. Figure 4.(A) and (B) show the typical $\mathrm{pH}$ image distribution in the microchannels with trap structure and the microchannels, respectively. The potential distribution of solution is related to the injection flow rate, and it is related to the reaction time of enzymatic reaction. Therefore, the kinetic process of enzymatic reaction can be described by the potential distribution of solution. Due to using OLED as the light, the resolution of LAPS maps was not well. 

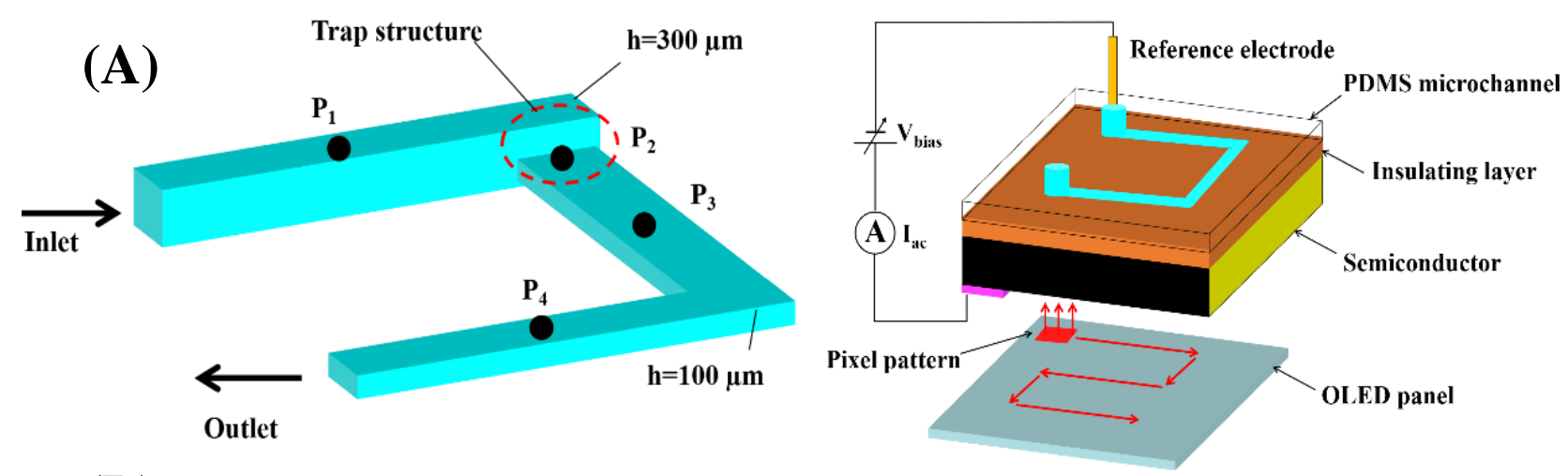

(B)

(a) $0.1 \mathrm{ml} / \mathrm{h}$

(b) $0.5 \mathrm{ml} / \mathrm{h}$
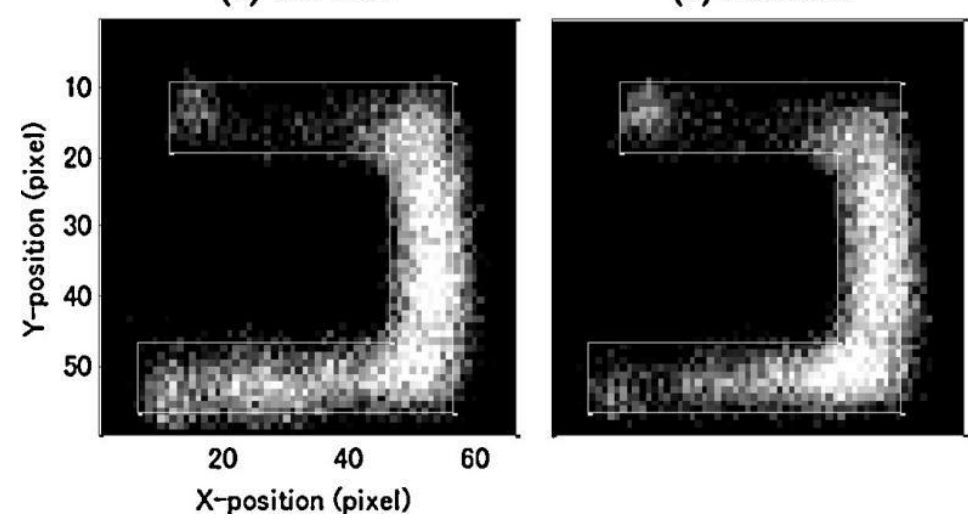

(c) $1.0 \mathrm{ml} / \mathrm{h}$

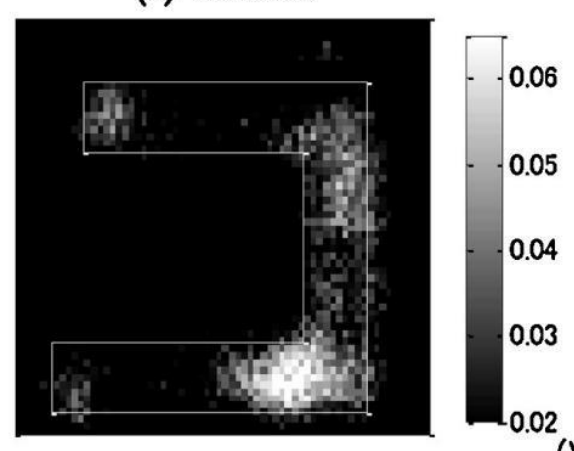

(V)

Figure 4.(A) Schematic Diagram of U-shaped Microchannel LAPS Analysis System with Trap Structure; (B) The distribution of enzymatic reaction images of urea solution in microchannels under different urease injection flow rates, (a) $0.1 \mathrm{ml} / \mathrm{h}$, (b) 0.5 $\mathbf{m l} / \mathbf{h},(\mathbf{c}) 1 \mathrm{ml} / \mathbf{h}^{[27]}$

LAPS analysis system can also realize visual analysis of neurotransmitters such as acetylcholine. Werner et al. constructed a miniature LAPS analysis system by modifying the surface of LAPS with acetylcholinase, and realized the visual analysis of acetylcholine ${ }^{[28]}$. Similarly, a CCD based $\mathrm{pH}$ image sensor can also be used for visual analysis of acetylcholine ${ }^{[29]}$.

\subsection{Microbial metabolism}

Metabolic perturbations of living bacterial and cells are essential for assessing bacterial and cellular activities, which can usually be measured by reflecting the rate of extracellular acidification. For instance, the activation of receptors can modulate the rate of extracellular acidification by causing metabolic changes that affect the demand for ATP. As a result, bacterial and cellular activities can be evaluated indirectly by measuring extracellular acidification. LAPS is one of the most commonly used measurement techniques of bacterial and cells metabolism in vivo, which is featured with an electrolyte-insulator-semiconductor (EIS) structure. When LAPS is illuminated by a modulated light source, it can generate an alternating photocurrent as a sensor signal. Traditional LAPS modified with a sensing layer of $\mathrm{Si}_{3} \mathrm{~N}_{4}$ has a pH sensitivity of about $57 \mathrm{mV} / \mathrm{pH}$. All these characteristics make LAPS an ideal device for the measurement of microbial metabolism, especially when it is integrated with microfluidic chips. For example, Liu Qingjun et al. reported a micro LAPS analysis system which can calculate the number of cells $^{[30]}$. The PDMS microchannel was prepared by micromachining technology, and the PDMS microchannel was bonded to the surface of LAPS to form a cross-shaped microchannel on silicon substrate. The cell culture medium was driven by peristaltic pump, and the rat blood cells driven by gravity were counted and analyzed by measuring LAPS photo-generated current. In this study, microfluidics technology is introduced into the research of LAPS cell sensor, which promotes the miniaturization and multifunction of cell counting analyzer.

In addition, LAPS chip has also been demonstrated to be suitable for monitoring cell activities stimulated by various drugs. For instance, Hu Ning et al. reported a LAPS analysis system for studying cellular metabolic 
mechanisms and drug reactions ${ }^{[31]}$. As shown in Figure 5, by using gravity to drive cell culture solution and taking LAPS as the substrate, the system constructs a microchannel cell culture chamber on its surface. The results show that the LAPS analysis system can detect cell metabolic activity in real time, and it is expected to become a practical platform for studying cell metabolic mechanism and drug evaluation.

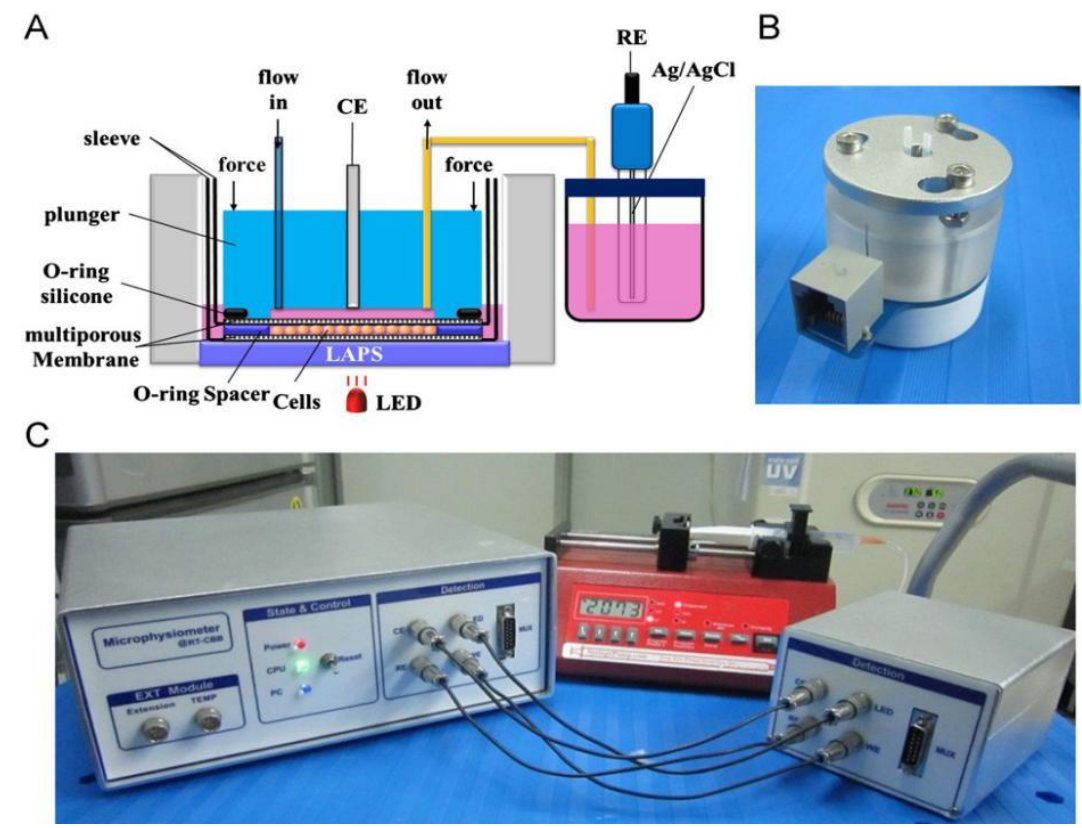

Figure 5.(A) A schematic structural diagram of cell microphysiology based on LAPS; (B) Microphysiometer Fluid Drive Unit; (C) Schematic diagram of the overall structure of the detection system ${ }^{[31]}$

Microfluidic chips can provide a stable micro environmental for living cells, which greatly facilitated cell culture and measurement. Liang Tao et al. reported a LAPS analysis system for detecting the metabolic rate of liver tumor cells ${ }^{[32]}$. As shown in Figure 6, by using a syringe pump to drive microfluidics and setting bubble capture structure in the microchannels, the interference of bubbles to the detection signal is effectively reduced. Since its modular structure and high scalability, the LAPS analysis system has a good application prospect in organ chip.

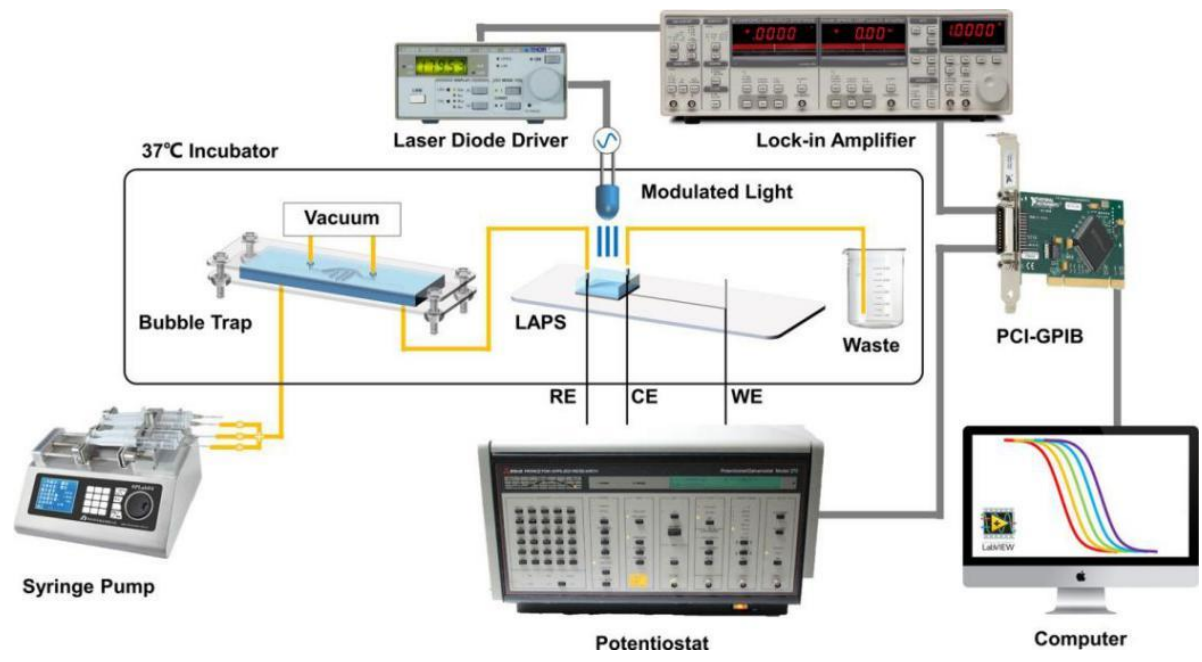

Figure 6 Schematic diagram of microchannels detection system based on LAPS ${ }^{[32]}$

The microfluidic chips are also integrated with LAPS chips to develop cell-based biosensors towards functional analysis of chemically sensitive cells. For example, Du Liping et al. reported a LAPS analysis system to detect the signal transduction mechanism of taste cells, which can realize the label-free functional analysis of bioengineering taste receptor cells via extracellular recording ${ }^{[33]}$. Extracellular potential changes of single bioengineered cells was recorded by LAPS. Microfluidic chip was capable of providing stable microenvironments for cell measurements 
with a well-defined concentration stimulus. As shown in Figure 7, LAPS can be used to detect the corresponding changes in cell membrane potential caused by specific bitter stimulation of taste cells. The LAPS analysis system is not only suitable for the determination of cells with specific functions, but also for the study of chemical signal transduction mechanism. However, this system was comparatively complex.

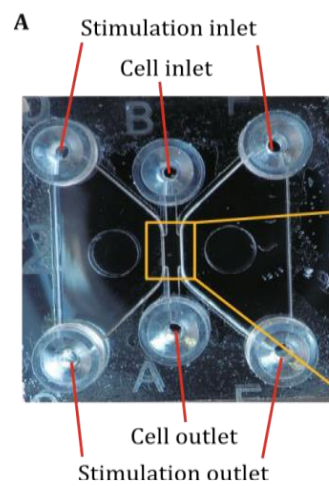

B
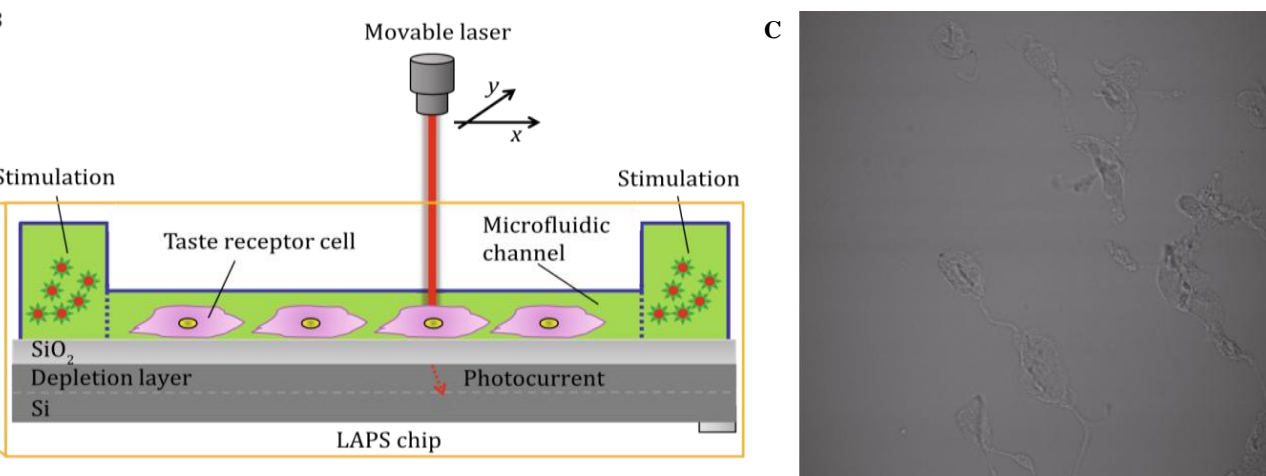

Figure 7.(A) A cell culture microanalysis system based on LAPS. The cell culture chip includes two stimulation microcavities and one cell culture microcavity; (B) The structure of the cell culture microcavity, which is combined with LAPS sensor to detect the change of membrane potential of taste receptor cells; (C) Cells in microfluidic channel ${ }^{[33]}$

In addition to cells, LAPS integrated microfluidic chip is also used to measure the organ metabolism cultured on the surface of LAPS chip. For example, Wu Qian et al. reported an organ-like chip system based on LAPS ${ }^{[34]}$. As shown in Figure 17, the circulation flow of culture solution in the organ-like chip was realized by means of microfluidics. Through LAPS detection system, the changes of metabolites in the microenvironment of organ culture solution were analyzed, so as to realize the real-time monitoring of the physiological state of similar organs.

\subsection{Digital droplet LAPS microanalysis system}

Digital droplet technology is an emerging one that greatly improves the capability of microfluidic chip to deal with the small volume solutions. It has also been applied to LAPS-based microanalysis systems. For instance, Miyamoto et al. reported a microdroplet analysis system based on LAPS ${ }^{[35]}$, as shown in Figure 8 . The system uses a microinjector to add analytical droplets to the sample inlet of the microchannels, closes the electronic valve, opens the peristaltic pump, and the analytical solution is attracted into the microchannels to generate a droplet. When the droplet suction inlet is in full contact with the reference electrode, the peristaltic pump is closed (the position of the droplet is controlled by the opening or closing of the valve. Its moving speed is about $0.78 \mathrm{~mm} / \mathrm{s}$ ). The microdroplets is completed by opening the LAPS detection device, in which the minimum volume generated by the microelectrochemical analysis system is $400 \mathrm{~nL}$. It is an excellent work to demonstrate the less solution consumed up to now, which still could decrease the volume of droplets and accuracy control them.

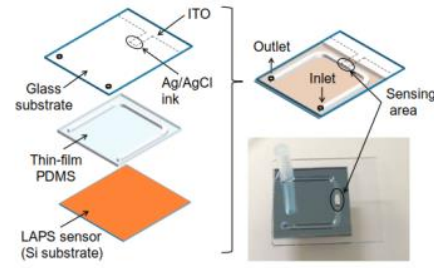

(a)

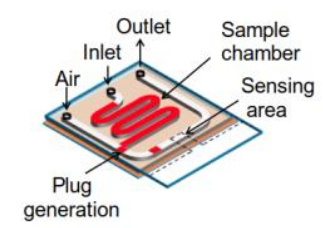

(c)

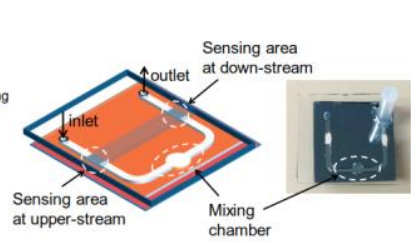

(b)

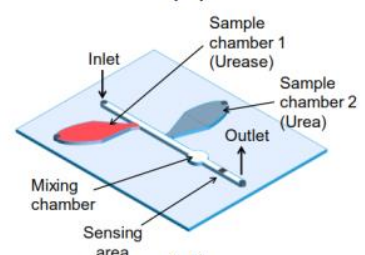

(d)

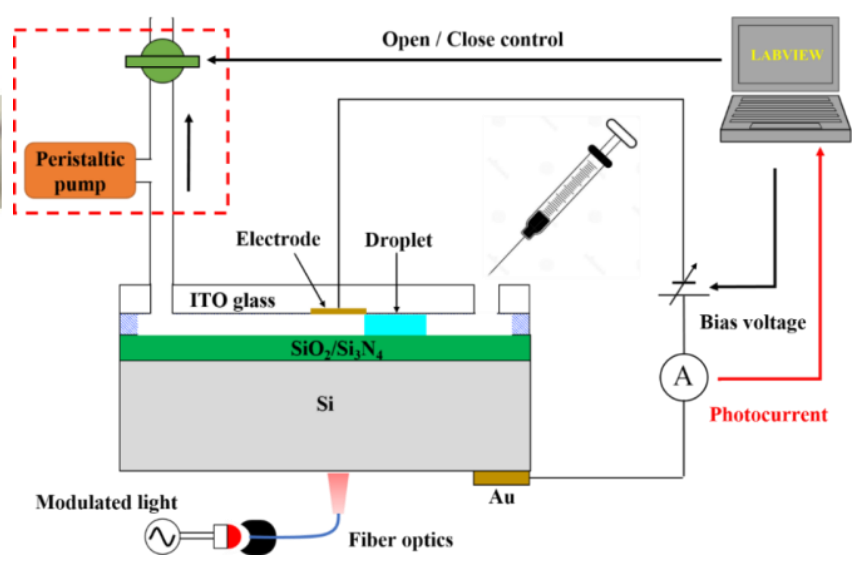

(e) 
Figure 8.(a) Test structure of the microfluidic device combined with LAPS; (b) Channel design with a chamber for merging and differential measurement; (c) Channel design to generate plugs on chip; (d) Test structure with two sample chambers, one merging chamber, and one sensing area; (e) Schematic diagram of a droplet analysis system based on LAPS ${ }^{[35]}$

To address the problems that the volume is large, the structure is complex, the integration is difficult and to complete the miniaturization process of the external pump, Li Xueliang et al. proposed the scheme to combine LAPS which is used as a microfluidics detection sensor with electroosmotic micropump to drive microfluidics ${ }^{[36]}$. The electroosmotic micropump doesn't have moving mechanical parts, and it is characterized by simple structure, easy embedding into microchannels, no pulsation, and accurate transportation of microfluidics, etc. Figure 9.(a) shows a schematic diagram of the structure and size of the microchannels. Figure 9.(b) and (c) show the top view and side view of the microchannels. T-shaped PDMS microchannels are constructed on the surface of LAPS. And the branch channel uses mechanical injection pump to transport the samples to be analyzed. Also an electroosmotic micropump is embedded in the main microchannels, while the deionized water is used as the working fluid, and there is an air gap between the working fluid and the sample. The working principles of the microelectrochemical analysis system are as follows: firstly, through the injection pump, the sample analysis solution is transported to the "D" intersection of the T-shaped microchannels, and then the injection pump is turned off; secondly, the electroosmotic micropump is started to generate electroosmotic flow which would move to the right, and the sample analysis solution at the " $\mathrm{D}$ " intersection would be pumped to the downstream of the main microchannels to generate a micro-droplet. Finally, when the micro-droplets reach the detection area of LAPS, the electroosmotic micropump is closed and the LAPS detection system is started to complete the micro-droplet detection.

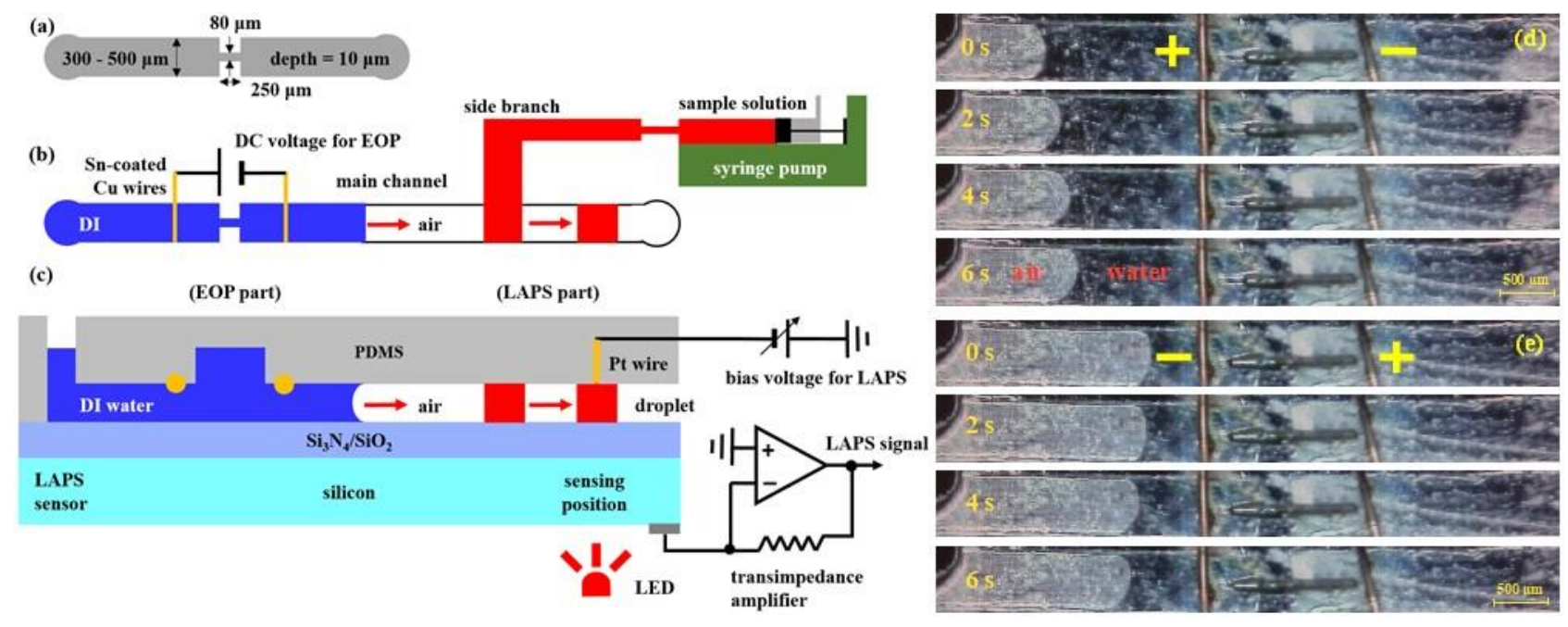

Figure 9.(a) Schematic diagram of microchannel structure and size; (b) and (c) a top view and a side view of the microchannels. The microelectrochemical analysis system includes an electroosmotic driving section and a LAPS detection section. A microdroplet is generated near the "D" intersection of T-shaped microchannels. The micro-droplet is pumped to the detection area of LAPS by electroosmotic micropump, and the micro-droplet contacts with Pt line to form a conduction loop to complete the detection of micro-droplet; EO flows in (d) right and (e) left directions ${ }^{[36]}$

In order to improve the infusion efficiency of electroosmotic micropump, Li Xueliang et al. proposed a technology of bubble-assisted electroosmotic micropump, and used it to construct a microelectrochemical analysis system $^{[37]}$. As shown in Figure 10, the microelectrochemical analysis system includes 3 sections-a bubble-assisted electroosmotic micropump section, a micro-droplet separation section, as well as a LAPS detection section. A microchannels with a width of $80 \mu \mathrm{m}$ and two Ti/Au electrodes (EO electrodes (right) and Droplet separator) are added in the downstream of the bubble-assisted electroosmotic micropump, which perform functions as Droplet separator and reference electrode respectively. The Droplet separator has the same function as the Bubble generator. A certain DC voltage is applied between EO electrode (right) and Droplet separator to generate bubbles through electrolysis, so as to separate a certain volume of micro-droplets. When the droplets are separated in the "bottleneck" 
area which is in the downstream of the microchannels, by bubble-assisted electroosmotic micropump, the microdroplets are pumped to the detection area of LAPS and contact with the reference electrode of LAPS to complete the droplets detection.

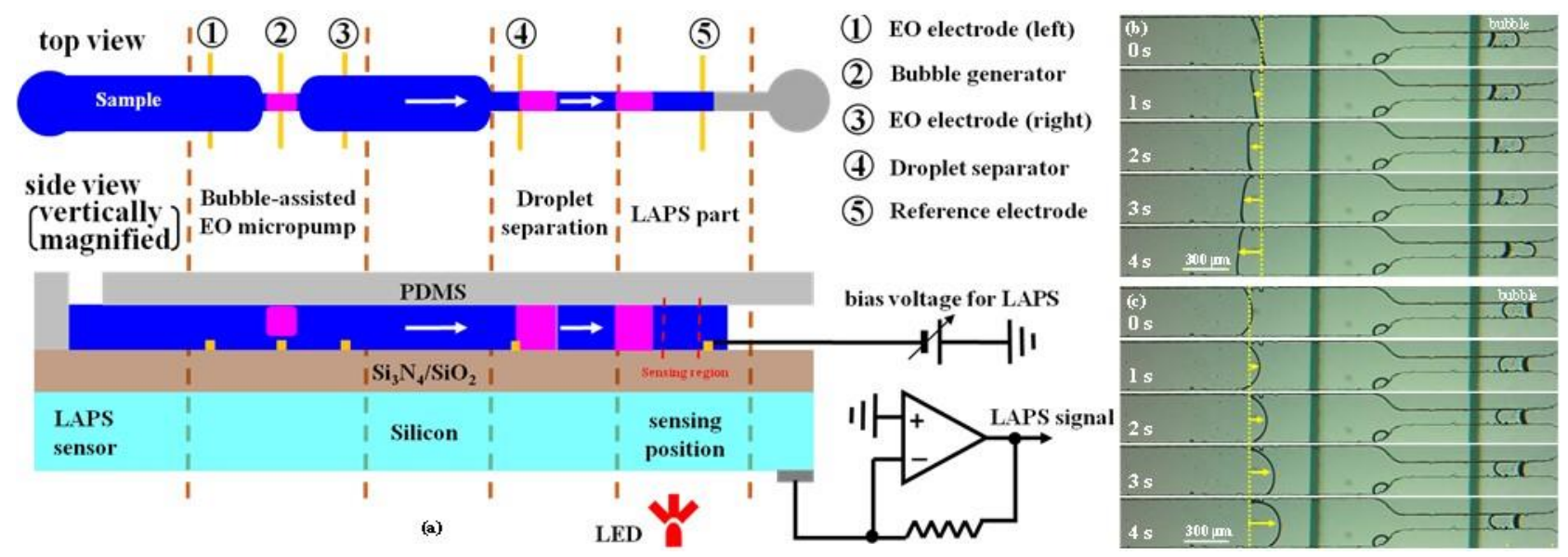

Figure 10.(a) Microelectrochemical analysis system with a LAPS as sensing unit and a bubble-assisted electroosmotic micropump as fluid driving unit; Bubble-assisted EO flows in (b) left and (c) right directions depending on the polarity of the pumping voltage ${ }^{[37]}$

\section{Conclusions and Prospects}

Since the birth of LAPS, it has been widely used in plenty of fields with its unique advantages. The research process of LAPS involves many disciplines such as chemistry, biology, semiconductor and electronic information, which is a typical interdisciplinary research. In order to promote the wide application of LAPS in biomedicine and other fields, in addition to improving the detection performance of sensors, the miniaturization of detection system is also very important. LAPS analysis system can not only reduce the consumption of biological and other valuable analysis samples, but also realize the rapid measurement of multi-parameters in the dynamic process of complex chemical and biological reactions. This paper reviews the applications of LAPS analysis system in ion diffusion, enzymatic reaction, microbial metabolic activity and droplet microfluidics.

At present, the LAPS analysis system has been demonstrated to be suitable for applications requiring high surface flatness of sensors or flexible definition of measurement areas, which show decisive advantages. However, LAPS analysis system still has some limitations that hinders its further development and practical application. More efforts should be devoted to improve the performances of LAPS analysis system to broaden their practical application fields. In the future, the development trend of LAPS analysis system will focus on the following aspects: (1) how to improve the spatial resolution of LAPS records. The possible solutions may come from using novel semiconductor materials to manufacture LAPS chips, introducing more focused light spots, and laterally suppressing the movement of carriers in semiconductors. (2) How to speed up the imaging system. To solve this problem, it is necessary to utilize a high-speed scanning system, which allows rapid recording of data and rapid imaging from the sensor surface. (3) How to improve the measurement accuracy of LAPS under the microfluidic conditions. Since the measurement in the micro or nanoscale solutions require very high accuracy, it is thus highly essential to improve the measurement accuracy of LAPS, especially in the cases of high-throughput measurement. The possible solution to this problem may rely on the improvement of the peripheral circuits and data processing algorithms. In summary, this paper needs to further improve the spatial resolution, measurement speed and accuracy of LAPS to promote the wide applications of LAPS analysis system in many fields such as biomedicine, food safety, and environmental protection ${ }^{[38-40]}$. 


\section{NOTES}

The authors declare that they have no conflicts of interest.

\section{FUNDING}

This work was financially supported in part by grants from the National Natural Science Foundation of China (grant numbers 51861145307, and 32071370) and is partly supported by the Key scientific research projects of colleges and universities in Henan Province (grand number 22B510022).

\section{References}

[1] G. M. Whitesides. The origins and the future of microfluidics [J]. Nature, 2006, 442: 368-373.

[2] T. Yoshinobu, K. Miyamoto, T. Wagner, et al. Recent developments of chemical imaging sensor systems based on the principle of the light-addressable potentiometric sensor[J]. Sensors \& Actuators B Chemical, 2015, 207: 926-932.

[3] D.G. Hafeman, J.W. Parce, H.M. McConnell. Light-addressable potentiometric sensor for biochemical systems [J]. Science, 1988, 240: 1182-1185.

[4] C. Yang, C. Chen, N. Akuli, et al. Chemical Illumination modification from an LED to a laser to improve the spatial resolution of IGZO thin film light-addressable potentiometric sensors in pH detections[J]. Sensors and Actuators B: Chemical, 2020, 329: 128953.

[5] Z. Wen, Y. Xu, H.E Tahir, et al. Rapid and wide-range determination of Cd(II), $\mathrm{Pb}(\mathrm{II}), \mathrm{Cu}(\mathrm{II}) \operatorname{and} \mathrm{Hg}(\mathrm{II})$ in fish tissues using light addressable potentiometric sensor [J]. Food Chemistry, 2016, 221: 541-547.

[6] W. Zhang, Y. Xu, X. Zou. Rapid determination of cadmium in rice using an all-solid RGO-enhanced light addressable potentiometric sensor[J]. Food Chemistry, 2018, 261: 1-7.

[7] W. Zhang, C. Liu, X. Zou, et al. Micrometer-scale light-addressable potentiometric sensor on an optical fiber for biological glucose determination[J]. Analytica Chemical Acta, 2020, 1123: 36-43.

[8] G. Li, W. Li, S. Li, et al. A novel aptasensor based on light-addressable potentiometric sensor for the determination of Alphafetoprotein[J]. Biochemical Engineering Journal, 2020, 164: 107780.

[9] J. Liang, N. Zhu, S. Li, et al. Light-addressable potentiometric sensor with gold nanoparticles enhancing enzymatic silver deposition for 1,5-anhydroglucitol determination[J]. Biochemical Engineering Journal, 2017, 123: 29-37.

[10] S. Dantism, S. Takenaga, P. Wagner, et al. Light-addressable Potentiometric Sensor (LAPS) Combined with Multi-chamber Structures to Investigate the Metabolic Activity of Cells[J]. Procedia Engineering, 2015, 120: 384-387.

[11] S. Dantism, S. Takenaga, T. Wagner, et al. Differential imaging of the metabolism of bacteria and eukaryotic cells based on lightaddressable potentiometric sensors[J]. Electrochemical Acta, 2017, 246: 234-241.

[12] C. Wu, L. Du, Y. Tian, et al. A Light-Addressable Potentiometric Sensor for Odorant Detection Using Single Bioengineered Olfactory Sensory Neurons as Sensing Element[J]. Methods in Molecular Biology, 2017, 1572: 233-246.

[13] C. Sda, A. Dr, A. Ts, et al. Quantitative differential monitoring of the metabolic activity of Corynebacterium glutamicum cultures utilizing a light-addressable potentiometric sensor system[J]. Biosensors and Bioelectronics, 2019, 139:111332-111332.

[14] L. Du, J. Wang, W. Chen, et al. Dual functional extracellular recording using a light-addressable potentiometric sensor for bitter signal transduction[J]. Analytica Chemical Acta, 2018, 1022: 106-112.

[15] C. Wu, T. Bronder, A. Poghossian, et al. Label-free detection of DNA using a light-addressable potentiometric sensor modified with a positively charged polyelectrolyte layer[J]. Nanoscale, 2015, 7(14): 6143-6150.

[16] Y. Jia, F. Li, T. Jia, et al. Meso-tetra(4-carboxyphenyl)porphine-Enhanced DNA Methylation Sensing Interface on a LightAddressable Potentiometric Sensor[J]. ACS Omega, 2019, 4(7):12567-12574.

[17] Y. Tian, T. Liang, P. Zhu, et al. Label-Free Detection of E. coli O157:H7 DNA Using Light-Addressable Potentiometric Sensors with Highly Oriented ZnO Nanorod Arrays[J]. Sensors, 2019, 19(24), 5473.

[18] Y. Guo, K. Miyamoto, T. Wagner, et al. Device simulation of the light-addressable potentiometric sensor for the investigation of the spatial resolution[J]. Sensors \& Actuators B Chemical, 2014, 204: 659-665.

[19] T. Wagner, K. Miyamoto, N. Shigihara, et al. Microfluidic systems with free definable sensor spots by an integrated light- 
addressable potentiometric sensor [J]. Procedia Engineering, 2011, 25: 791-794.

[20] T. Yoshinobu, K. Miyamoto, C.F. Werner, et al. Light-Addressable Potentiometric Sensors for Quantitative Spatial Imaging of Chemical Species[J]. Annual Review of Analytical Chemistry , 2017, 10(1): 225-246.

[21] M. Nakao, T. Yoshinobu, H. Iwasaki. Scanning-laser-beam semiconductor pH-imaging sensor [J]. Sensors and Actuators B, 1994, 20: $119-123$.

[22] T. Liang, Y. Qiu, Y. Gan, et al. Recent Developments of High-Resolution Chemical Imaging Systems Based on Light-Addressable Potentiometric Sensors (LAPSs)[J]. Sensors, 2019, 19(19): 1-22.

[23] T. Yoshinobu, T. Harada, H. Iwasaki. Application of the pH-Imaging Sensor to Determining the Diffusion Coefficients of Ions in Electrolytic Solutions [J]. Japanese Journal of Applied Physics, 2000, 39: 318-320.

[24] T. Yoshinobu, H. Iisaki, Y. Ui, et al. The light-addressable potentiometric sensor for multi-ion sensing and imaging [J]. Methods, 2005, 37: 94-102.

[25] K. Miyamoto, H. Ichimura, T. Wagner, et al. Chemical imaging of the concentration profile of ion diffusion in a microfluidic channel [J]. Sensors and Actuators B, 2013, 189: 240-245.

[26] K. Miyamoto, A. Itabashi, T. Wagner, et al. High-speed chemical imaging inside a microfluidic channel [J]. Sensors and Actuators B, 2014, 194: 521-527.

[27] K. Miyamoto, M. Yoshida, T. Sakai, et al. Differential Setup of Light-Addressable Potentiometric Sensor with an Enzyme Reactor in a Flow Channel [J]. Japanese journal of applied physics, 2011, 50: 1-5.

[28] C.F. Werner, S. Takenaga, H. Taki, et al. Comparison of label-free ACh-imaging sensors based on CCD and LAPS [J]. Sensors and Actuators B, 2013, 177:745-752.

[29] S. Takenaga, Y. Tamai, K. Okumura, et al. Label-Free Acetylcholine Image Sensor Based on Charge Transfer Technology for Biological Phenomenon Tracking [J]. Japanese Journal of Applied Physics, 2012, 51: 1-5.

[30] Q.J. Liu, N. Hu, W.W. Ye, et al. Sensor chip for cell counting and analyzing [J]. Chinese journal of sensors and actuators, 2010, 23: 753-756. (in Chinese)

[31] N. Hu, C. Wu, D. Ha, et al. A novel microphysiometer based on high sensitivity LAPS and microfluidic system for cellular metabolism study and rapid drug screening [J]. Biosensors and Bioelectrons, 2013, 40: 167-173.

[32] T. Liang, C.L. Gu, Y. Gan, et al. Microfluidic chip system integrated with light addressable potentiometric sensor (LAPS) for real-time extracellular acidification detection [J]. Sensors and Actuators B, 2019, 301: 1-8.

[33] L.P. Du, W. Chen, Y. Tian, et al. A light-addressable microfluidic device for label-free functional assays of bioengineered taste receptor cells via extracellular recording [J]. 2019, 5: 73-79.

[34] Q. Wu, Y.X. Pan, H. Wan, et al. Research progress of organoids-on-chips in biomedical application. Chin Sci Bull, 2019, 64: 902910. (in Chinese)

[35] K. Miyamoto, S. Takuya, A. Minami, et al. Light-Addressable Potentiometric Sensor as a Sensing Element in Plug-Based Microfluidic Devices [J]. Micromachines, 2016, 7: 111-118.

[36] X.L. Li, S.B. Liu, J.T. Liang, et al. Application of electroosmotic micropumps to a microfluidic system combined with a lightaddressable potentiometric sensor [J]. Physica status solidi (a), 2016, 213: 1500-1504.

[37] X.L. Li, S.B. Liu, P.P. Fan, et al. A bubble-assisted electroosmotic micropump for a delivery of a droplet in a microfluidic channel combined with a light-addressable potentiometric sensor [J]. Sensors and Actuators B, 2017, 248: 993-997.

[38] X. Yan, D. Chen, X. Tong. Light addressable potentiometric sensor phase rapid detection method based on orthogonal detection[C]. Eleventh International Conference on Information Optics and Photonics (CIOP 2019). 2019.

[39] Y. Tu, N. Ahmad, J. Briscoe, et al. Light-Addressable Potentiometric Sensors Using ZnO Nanorods as the Sensor Substrate for Bioanalytical Applications[J]. Analytical Chemistry, 2018, 90(14): 8708-8715.

[40] F. Li, J. Zhang, S. Hu, et al. Possibility of Combining Carbon Dots and Liquid Exfoliated Graphene as a Carbon-Based Light Addressable Potentiometric Sensor[J]. ACS Sensors, 2021, 6(3): 1218-1227. 